\title{
Kaempferol and Its Glycoside, Kaempferol 7-O-rhamnoside, Inhibit PD-1/PD-L1 Interaction In Vitro
}

\author{
Ji Hye Kim ${ }^{1}{ }^{1}$, Young Soo Kim ${ }^{1}$, Jang-Gi Choi ${ }^{1}$, Wei Li ${ }^{1}{ }^{\circledR}$, Eun Jin Lee ${ }^{1}$, Jin-Wan Park ${ }^{2}$, \\ Jaeyoung Song ${ }^{2}$ and Hwan-Suck Chung ${ }^{1, *(D)}$ \\ 1 Korean Medicine (KM)-Application Center, Korea Institute of Oriental Medicine (KIOM), Dong-gu, \\ Daegu 41062, Korea; jkim2903@kiom.re.kr (J.H.K.); yskim527@kiom.re.kr (Y.S.K.); \\ jang-gichoi@kiom.re.kr (J.-G.C.); liwei1986@kiom.re.kr (W.L.); dldmswls0416@naver.com (E.J.L.) \\ 2 New Drug Development Center, Daegu Gyeongbuk Medical Innovation Foundation (DGMIF), Dong-gu, \\ Daegu 41061, Korea; jinwanpark@dgmif.re.kr (J.-W.P.); jysong@dgmif.re.kr (J.S.) \\ * Correspondence: hschung@kiom.re.kr; Tel.: +82-53-940-3875
}

Received: 6 April 2020; Accepted: 29 April 2020; Published: 3 May 2020

\begin{abstract}
Kaempferol (KO) and kaempferol 7-O-rhamnoside (KR) are natural products from various oriental herbs such as Geranii Herba. Previous studies have reported some biological activities of $\mathrm{KO}$ and $\mathrm{KR}$; however, their effects on PD-1/PD-L1 interaction have not been reported yet. To elucidate their inhibitory activities on PD-1/PD-L1 protein-protein interaction (PPI), biochemical assays including competitive ELISA and biolayer interferometry (BLI) systems were performed. Cellular PD-1/PD-L1 blocking activity was measured in a co-culture system with PD-1 Jurkat and PD-L1/aAPC CHO-K1 cells by T-cell receptor (TCR) activation-induced nuclear factor of activated T cells (NFAT)-luciferase reporter assay. The detailed binding mode of action was simulated by an in silico docking study and pharmacophore analysis. Competitive ELISA revealed that $\mathrm{KO}$ and its glycoside KR significantly inhibited PD-1/PD-L1 interaction. Cellular PD-1/PD-L1 blocking activity was monitored by $\mathrm{KO}$ and KR at non-cytotoxic concentration. Surface plasmon resonance (SPR) and biolayer interferometry (BLI) analysis suggested the binding affinity and direct inhibition of KR against PD-1/PD-L1. An in silico docking simulation determined the detailed mode of binding of KR to PD-1/PD-L1. Collectively, these results suggest that KR could be developed as a potent small molecule inhibitor for PD-1/PD-L1 blockade.
\end{abstract}

Keywords: programmed cell death protein 1; programmed death-ligand 1; kaempferol; kaempferol 7-O-rhamnoside; kaempferitrin; immune checkpoint inhibitor; small molecule inhibitors

\section{Introduction}

Programmed cell death protein 1 (PD-1) is one of the immune checkpoints and is known to be involved with immunological self-tolerance [1,2]. Various immune cells, including activated T cells, have been reported to express PD-1 [2,3]. Thus, PD-1 plays a suppressive role toward T cell activity in peripheral tissues and in the tumor microenvironment [4].

Cytokines from the tumor microenvironment such as interferon-gamma (IFN- $\gamma$ ) induce PD-1 ligand-1 (PD-L1), and the PD-1 ligands are found on most cancers. Interaction of PD-1 and PD-L1 is closely associated with impaired $\mathrm{T}$ cell functions [5], allowing cancer cells to escape immune surveillance [6,7]. Therefore, reversal of $\mathrm{T}$ cell dysfunction through blocking of PD-1/PD-L1 is considered an important strategy for enhancing immunity to cancer.

In recent decades, it has been thought that an ideal strategy for cancer immunotherapy would be an immune checkpoint blockade (ICB) targeting the PD-1/PD-L1 pathway [8]. Most of these inhibitors are 
monoclonal antibodies; however, some limitations have been reported, including high costs for patients, restricted tumor burden penetration and unresponsiveness of ICB therapy [9]. To overcome these shortcomings, small molecules with low molecular weight have gained interest across chemical-based, and peptide-based checkpoint inhibitors.

Natural substances have been reported to possess potential for diverse biological activities with various molecular mechanisms [10-13]. Notably, recent studies have been focused on modulators of the PD-1/PD-L1 pathway to suppress development and progression of cancers [14-17]. Recently, several studies have reported that phenolic compounds from Glycyrrhiza uralensis [18] and caffeoylquinic acid compounds [19] showed inhibitory activities on PD-1/PD-L1 protein-protein interaction (PPI) $[18,19]$. Therefore, traditional herbal medicinal resources have possessed extensive potential as immune checkpoint modulators for immunotherapeutic agents.

The present study found that Geranii Herba extract (GHE) is a novel candidate agent for PD-1/PD-L1 inhibition. GHE was reported to contain various phytochemicals including flavonoids and phenolic compounds [20,21]. Among them, kaempferitrin (KI, kaempferol-3,7-dirhamnoside) was identified as one of the abundant compounds of GHE in our previous reports [22]. Interestingly, KI has been known to be hydrolyzed to kaempferol (KO) and kaempferol 7-O-rhamnoside (KR) in the human intestine by the gut microbiome [23]. In addition, $\mathrm{KO}$ was generated by enzymatic hydrolysis with $\alpha$-L-rhamnoside and/or $\beta$-glycosidase from KI and KR in vitro [24].

Previous studies on $\mathrm{KO}$ and $\mathrm{KO}$ rhamnosides have reported diverse biological activities, including anti-oxidant [25], anti-inflammatory [24], and anti-tumor activities [26]. Although they have been widely examined, their PD-1/PD-L1 blockade effects have not yet been studied; to the best of our knowledge, this study is the first to describe their potential for PD-1/PD-L1 inhibition.

\section{Results}

\subsection{Effects of $K O$ and Its Glycosides on PD-1/PD-L1 Protein Interaction}

To elucidate a potent candidate agent as a PD-1/PD-L1 interaction inhibitor, the effect of GHE, which contains $\mathrm{KO}$ and its glycosides, KR and KI (Figure 1), was examined using a competitive ELISA according to a previous study [27]. As a positive control, PD-1 or PD-L1 neutralizing antibody ( $\alpha$ PD-1 or $\alpha$ PD-L1) and small molecule PD-1/PD-L1 inhibitor C1 were used (Figure 2A-C). The result showed that GHE dose-dependently inhibited PD-1 and PD-L1 protein-protein interaction (PPI) at an $\mathrm{IC}_{50}$ value of $87.93 \mu \mathrm{g} / \mathrm{mL}$ (Figure S1). To determine which active compounds of GHE have inhibitory effects on PD-1/PD-L1 interaction, a comparison study was performed. As shown in Figure 2D, KO showed the best blocking effect with an $\mathrm{IC}_{50}$ of $7.797 \mu \mathrm{M}$. KR and $\mathrm{KI}$ also revealed inhibitory effects on PD-1/PD-L1 binding but did not show dose-dependent activities. These results indicated that the active compounds of GHE on PD-1/PD-L1 blockade may be KO and its glycosyl compounds.

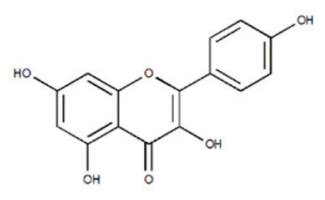

Kaempferol

(KO)

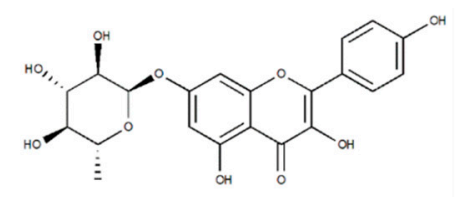

Kaempferol 7-0-rhamnoside (KR)

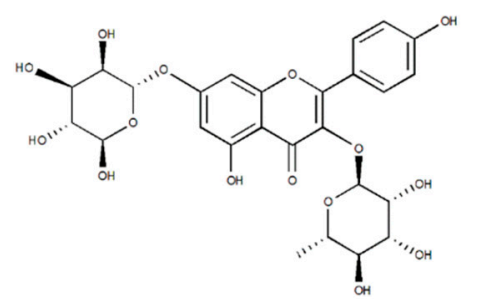

Kaempferol-3,7-dirhamnoside (Kaempferitrin, KI)

Figure 1. The chemical structures of kaempferol (KO), kaempferol 7-O-rhamnoside (KR), and kaempferol-3,7-dirhamnoside (kaempferitrin, KI). Chemical structures were generated using ChemDraw Professional 8.0. 
A

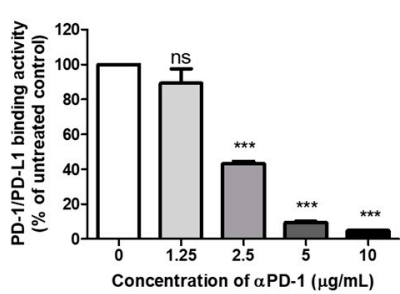

D

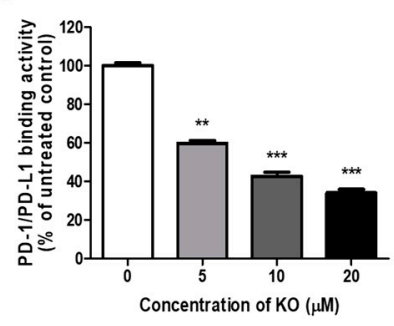

B

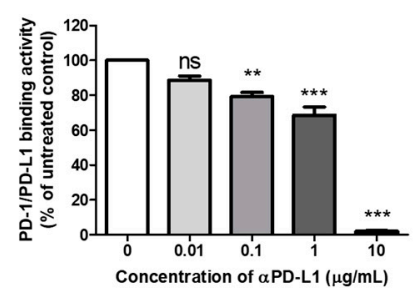

E

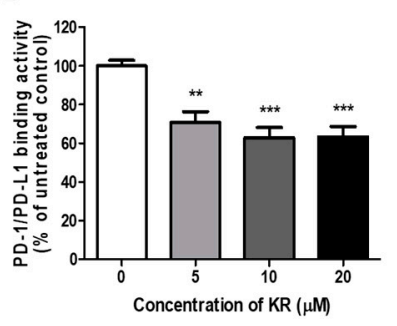

C

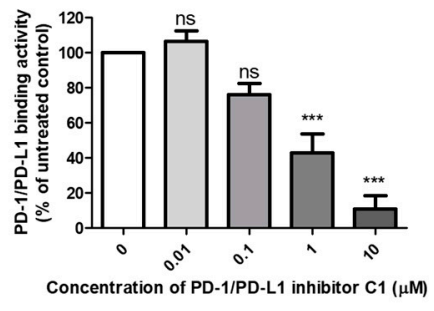

$\mathbf{F}$

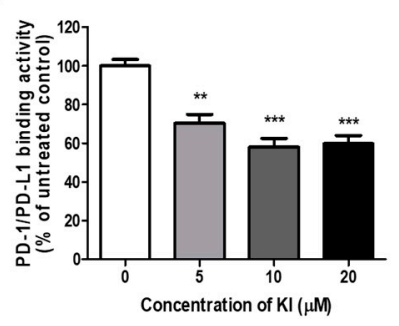

Figure 2. Effects of $\mathrm{KO}$ and its glycosides on programmed cell death protein 1 (PD-1)/PD-1 ligand-1 (PD-L1) protein interaction in a competitive ELISA. (A) PD-1 neutralizing antibody, (B) PD-L1 neutralizing antibody, (C) PD-1/PD-L1 inhibitor C1, (D) KO, (E) KR, and (F) KI were pre-treated onto plates coated with PD-L1, followed by incubation with biotinylated PD-1. Relative PD-1/PD-L1 binding activities were determined using a competitive ELISA assay, as described in the Materials and Methods. Data are presented as means \pm S.E. (standard error) values of three independent experiments. Asterisks indicate significant inhibition of PD-1/PD-L1 binding activity by each test inhibitor as compared with the control group. (** $p<0.01$, and ${ }^{* *} p<0.001$; ns: not significant).

\subsection{Effects of $K O$ and Its Glycosides on PD-1/PD-L1 Interaction in a Cell Model System}

It has been widely reported that the PD-1/PD-L1 axis is closely related to T cell function, and the reversal of $\mathrm{T}$ cell dysfunction has been suggested as an effective immune therapeutic strategy against cancer [28-30]. To screen and evaluate inhibitors for the PD-1/PD-L1 blockade, the effects of KO and its glycosides were investigated using the PD-1/PD-L1 blockade bioassay system [31,32]. In this system, two cell model systems were utilized; immortalized human $\mathrm{T}$ lymphocyte cells (Jurkat cells) were altered to constitutively express PD-1 and a T-cell receptor (TCR)-inducible nuclear factor of activated T cells (NFAT)-luciferase reporter (PD-1 Jurkat T cells), and CHO-K1 cells were modified to stably express human PD-L1 and TCR agonist (PD-L1/aAPC CHO-K1 cells) for production of antigen-presenting surrogate $\mathrm{CHO}$ cells [8]. First, to exclude the cytotoxic effect of $\mathrm{KO}$ and its glycosides on each cell model system, a Cell Counting Kit-8 (CCK) assay was conducted (Figure S2). Results showed that all of the compounds (KO, KR, and KI) were not cytotoxic up to $100 \mu \mathrm{M}$ in either cell line. Therefore, subsequent experiments were performed at the observed non-cytotoxic concentrations.

When co-cultured with PD-1 Jurkat T cells and PD-L1/aAPC CHO-K1 cells, TCR activation is restrained by PD-1/PD-L1 ligation and the NFAT-luciferase reporter activity. When PD-1/PD-L1 interaction is disrupted, TCR activation induces luminescence via activation of the NFAT pathway in co-culture systems [32]. Therefore, the effects of $\mathrm{KO}$ and its glycosides on PD-1/PD-L1 interaction in the cell model system were examined by measuring NFAT-luciferase reporter activity after co-culture with PD-1/NFAT Reporter-Jurkat cells and PD-L1/aAPC CHO-K1 cells. Results exhibited that KO and KR blocked PD-1/PD-L1 interaction via NFAT transcriptional activity in a dose-dependent manner (approximately 3 to 3.5 fold) (Figure 3A,B). There were no significant differences between $\mathrm{KO}$ and $\mathrm{KR}$ with $\mathrm{EC}_{50}$ values of 16.46 and $15.37 \mu \mathrm{M}$, respectively. On the other hand, KI had no effect at the indicated concentration (Figure $3 \mathrm{C}$ ). These results confirmed the potential blocking ability of $\mathrm{KO}$ and $\mathrm{KR}$ as a small molecule inhibitor of PD-1/PD-L1 interaction in cell model systems, but not upregulation of PD-1 level (Figure S3). The results described above indicate that $\mathrm{KO}$ and its glycosides are involved with the PD-1/PD-L1 blockade, as confirmed by the biochemical assay. 


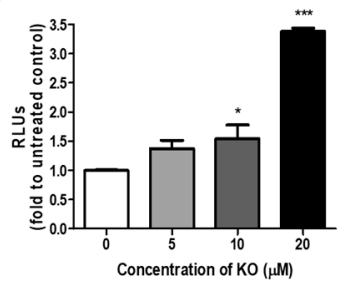

C

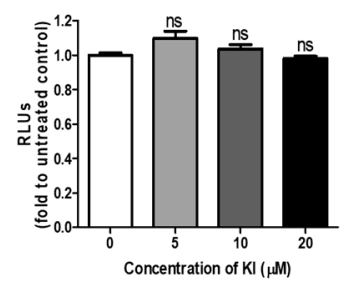

B

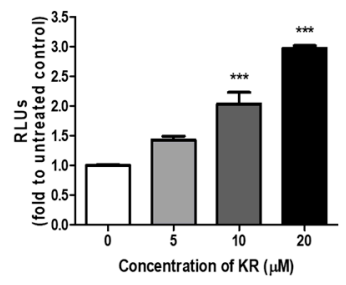

D

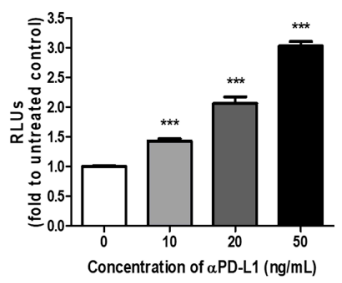

Figure 3. Effects of $\mathrm{KO}$ and its glycosides in cell-based PD-1/PD-L1 blockade assay. Effect of (A) KO, (B) KR, (C) KI and (D) aPD-L1 on T-cell receptor (TCR)-mediated nuclear factor of activated T cells (NFAT) activity by the PD-1/PD-L1 blockade in a co-cultured system. PD-1 Jurkat effector cells and PD-L1/aAPC CHO-K1 target cells were co-cultured with each compound for $24 \mathrm{~h}$ at the indicated concentrations. The activation of PD-1 Jurkat cells led to the level of NFAT-luciferase (for details, see Materials and Methods). Data are presented as means \pm S.E. (standard error) values of three representative independent experiments. Asterisks indicate significant upregulation of relative luminescence units (RLUs) by each sample as compared with the control group $\left({ }^{*} p<0.05\right.$ and *** $p<0.001$; ns: not significant).

\subsection{The Binding Interaction and Affinity of KR with PD-1 and PD-L1}

In order to measure the binding affinity of KR to PD-1 and PD-L1, BLI analysis was conducted in accordance with a previous study [33]. An increase of KR concentration revealed a wavelength shift in the BLItz sensorgram, indicating that KR was more bound to biotinylated PD-1 (Figure 4A) and PD-L1 (Figure 4B), respectively, when immobilized on the streptavidin (SA) sensor. The equilibrium dissociation constants $\left(\mathrm{K}_{\mathrm{D}}\right)$ of $\mathrm{KR}$ to PD-1 and PD-L1 were $3.11 \times 10^{-5}$ and $1.97 \times 10^{-5} \mathrm{M}$, with coefficients of determination, $\mathrm{R}^{2}$, of 0.9925 and 0.9958 , respectively. Although KR displayed a slightly higher $K_{D}$ value to PD-L1 than PD-1, it seems the affinities of KR to PD-1 and PD-L1 were comparable based on the results (Table 1). Additionally, to validate whether the molecular target of KR is PD-1 or PD-L1, SPR analysis was performed using Biacore T200 (GE Healthcare, Chicago, IL, USA) equipment according to a previous study [19]. Results showed that KR binds with recombinant PD-1 with a $K_{D}$ value of $1.56 \times 10^{-4} \mathrm{M}$ (Figure $\mathrm{S4}$ ); however, the $\mathrm{K}_{\mathrm{D}}$ value of $\mathrm{KR}$ with PD-L1 was not determined. Unexpectedly, $\mathrm{KO}$ was not analyzed in the BLItz and SPR analyses; this may be due to its low water solubility. Overall, these results suggested that KR possessed the potential to block activity against the PD-1/PD-L1 interaction by directly targeting PD-1 and PD-L1.

Table 1. The kinetic analysis of KR binding to PD-1 and PD-L1 using BLItz system.

\begin{tabular}{ccccc}
\hline Protein & $\boldsymbol{K}_{\mathbf{D}}(\mathbf{M})$ & $\boldsymbol{k}_{\mathbf{a}}\left(\mathbf{M}^{-\mathbf{1}} \mathbf{s}^{-\mathbf{1}}\right)$ & $\boldsymbol{k}_{\mathbf{d}}\left(\mathbf{s}^{-\mathbf{1}}\right)$ & $\boldsymbol{R}^{\mathbf{2}}$ \\
\hline PD-1 & $3.11 \times 10^{-5}$ & $3.15 \times 10^{2}$ & $9.79 \times 10^{-3}$ & 0.9925 \\
PD-L1 & $1.97 \times 10^{-5}$ & $3.92 \times 10^{2}$ & $7.73 \times 10^{-3}$ & 0.9958 \\
\hline
\end{tabular}


A

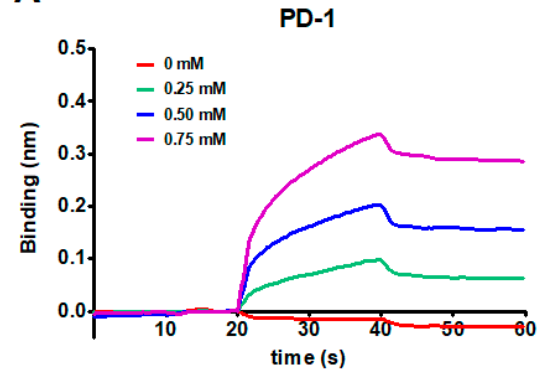

B

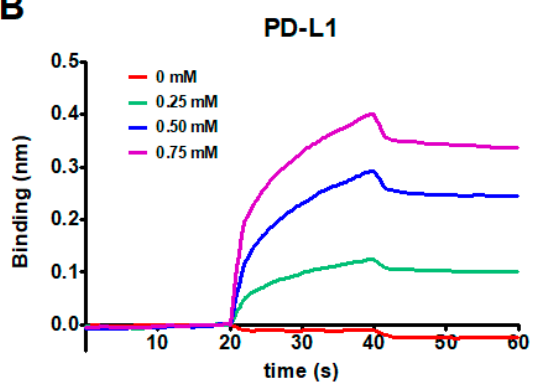

Figure 4. Global kinetic analysis of KR binding to biotinylated PD-1 (A) and PD-L1 (B) immobilized on a streptavidin biolayer interferometry (BLI) sensor. The binding of test inhibitors with PD-1 was confirmed by BLI analysis. The kinetics of KR for immobilized PD-1 and PD-L1 were monitored with increasing concentrations of $\mathrm{KR}(0,0.25,0.5$, and $0.75 \mathrm{mM})$ dissolved in phosphate-buffered saline (PBS) buffer ( $\mathrm{pH} 7.3$ ) containing 1\% DMSO. Data are presented as representative of three independent experiments.

\subsection{KO and Its Glycosides Inhibit PD-1/PD-L1 Protein Interaction In Silico}

To elucidate the detailed binding modes of $\mathrm{KO}$ and its glycosides with PD-1/PD-L1, binding energies obtained from molecular docking simulation were elucidated by in silico modeling (Figure 5). $\mathrm{KO}$ and its glycosides attached to the PD-L1 region at the site of PD-1, but the detailed mode of action was different. The predicted binding energy of PD-1 to KO was $-5.4 \mathrm{kcal} / \mathrm{mol}$, followed by $-5.6 \mathrm{kcal} / \mathrm{mol}$ of KR (Figure 5A, left panel). In the case of PD-L1, it was $\mathrm{KO}$ for $-5.0 \mathrm{kcal} / \mathrm{mol}$ and KR for $-5.3 \mathrm{kcal} / \mathrm{mol}$, respectively (Figure $5 \mathrm{~A}$, right panel). In addition, all $\mathrm{KO}$ and its glycosides showed a slightly lower or similar binding affinity to that of PD-1/PD-L1 inhibitor C1 (Figure 5A and Table S1). These results theoretically confirmed that KR has a higher possibility of binding with PD-1 and/or PD-L1 compared to KO.

A

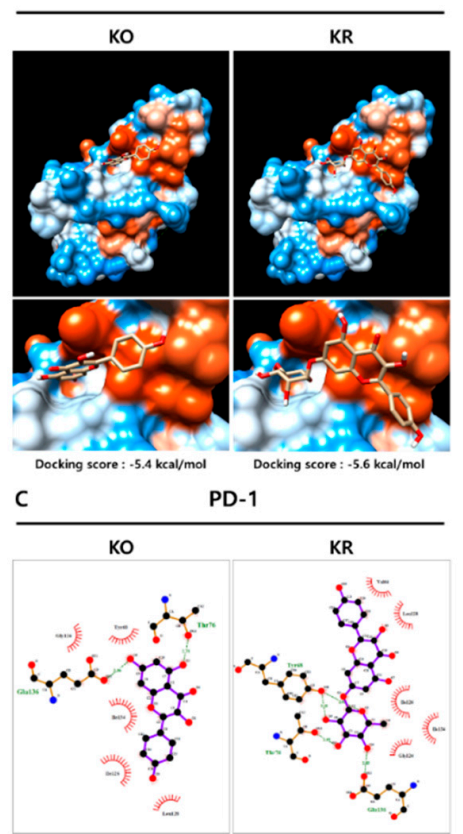

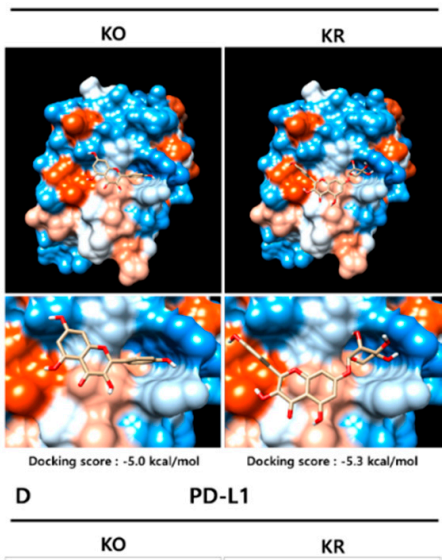

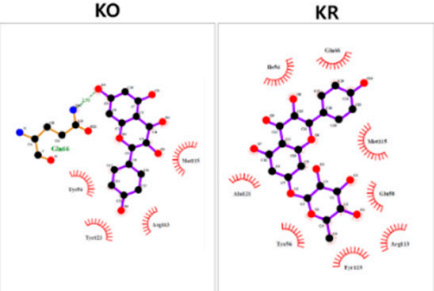

Figure 5. Hypothetical model of human PD-L1 or PD-1 in complex with KR. (A,B) Protein-ligand docking simulation for PD-1 (A) or PD-L1 (B) with each compound. Binding models were obtained through docking simulation derived from the PD-1/PD-L1 complex (PDB code 4ZQK) using AutoDock Vina. (C,D) Pharmacophore analysis of PD-1 (C) or PD-L1 (D). The hydrogen bonds and hydrophobic interactions between PD-L1/PD-1 and test inhibitors were analyzed using the LigPlot+ program. 
According to a previous study [34], the PD-1/PD-L1 complex is generated via major interaction with critical residues of PD-1 (Va164, Ile126, Leu128, Ala132, Ile134) and PD-L1 (Ile54, Tyr56, Met115, Ala121, Tyr123). Based on this information, pharmacophore analysis was represented as a 2D interaction diagram of PD1 or PD-L1 with each compounds (Figure 5B). Carbon at the 7 position in the glycosyl group of KR interacted with hydrophobic residues of Gly124, Ile126, and Ile134 (red crown mark) at the PD-L1 binding site of PD-1 protein (Table S2). Additionally, the glycoside group generated a hydrogen bond with residues Tyr68, Thr76, and Glu136 (green dashed line) at the PD-L1 binding site of the PD-1 protein. The hydroxyl group in positions at carbon 18 and 20 formed the interaction with residue Val64 (red crown mark), and that at carbon 15 interacted with Lue128 at the PD-L1 binding site of PD-1 protein. Therefore, major amino acids of KR for binding to PD-1 were identified as four residues: Val64, Ile126, Leu128, and Ile134. Collectively, we speculated that the glycoside group might be closely associated with the functional activity of KR in the PD-1/PD-L1 interaction.

\section{Discussion}

In recent decades, extensive developments have been achieved to develop cancer agents for cancer immunotherapy. Studies to develop small molecule inhibitors for relatively inexpensive cancer therapeutics are in progress [2]. CA-170 (also known as AUPM170 or PD-1-IN-1) and Aurigene-1 have been reported as potent small molecule PD-L1 antagonists; however, biochemical results suggested that they did not target hPD-L1 directly but may be mediated by another mode of action $[35,36]$. These cases emphasize the importance of detailed mechanisms based on evaluation methods from biochemical assay to in vivo assay. Therefore, careful development of small molecules for targeting the PD-1/PD-L1 immune checkpoint with the proper molecular mode of action is required.

Based on previous studies, $\mathrm{KO}$ and its glycosides have been reported to possess diverse pharmacological activities such as anti-oxidant [25], anti-inflammatory [37], and anti-virus activities [22]. To the best of our knowledge, the present study initially reported that $\mathrm{KO}$ and KR possess PD-1/PD-L1 inhibitory activity in vitro and in silico. KI was the most abundant component of GHE; however, only hydrolysate forms of $\mathrm{KI}(\mathrm{KO}$ and $\mathrm{KR})$ were effective in this study. These results suggest that $\mathrm{KO}$ and $\mathrm{KR}$, as bioactive compounds of GHE, interfere with PD-1/PD-L1 interaction.

In silico modeling and pharmacophore assays were performed to elucidate the more detailed binding mode of KR and the PD-1/PD-L1 complex (Figure 5). Using the AutoDock Vina program, a docking study calculated the binding affinity of the PD-1/PD-L1 complex (Figure 5, Tables S1 and S2). These results were experimentally confirmed using BLI analysis (Figure 4), and the binding affinities of KR toward hPD-1 and hPD-L1 were confirmed as $3.11 \times 10^{-5}$ and $1.97 \times 10^{-5} \mathrm{M}$, respectively. In a previous study, Mazewski et al. reported that some small molecule inhibitors of PD-1/PD-L1 developed by Bristol-Myers Squibb (BMS) Company showed high $\mathrm{K}_{\mathrm{d}}$ values ranging from $\mu \mathrm{M}$ to $\mathrm{mM}$ [38].

In the case of $\mathrm{KO}$, similar to the previous report [38], KO could not be analyzed because of the limits of solubility and fastening of the peak. It was reported that $\mathrm{KO}$ and $\mathrm{KR}$ are slightly soluble in water, with a solubility of $440 \mathrm{mg} / \mathrm{L}(1.537 \mathrm{mM})$ for $\mathrm{KO}$ and $1.16 \mathrm{mg} / \mathrm{mL}(2.68 \mathrm{mM})$ for KR. Most of the kaempferol in plants contains glycoside moieties rather than in the free form, and it has been reported to affect bioavailability and bioactivity [39]. A previous study reported that KI is known to be hydrolyzed to $\mathrm{KO}$ and KR in the human intestine by the gut microbiome [40]. Interestingly, the present study confirmed that KI derivatives (KO and KR) generated by deglycosylation showed more potent PD-1/PD-L1 inhibitory activity in vitro (Figures 2-4) and in silico (Figure 5), indicating potential for PD-1/PD-L1 interaction inhibitors. However, considering that $\mathrm{KO}$ reaches $35 \mathrm{nM}$ to $1.61 \mu \mathrm{M}$ concentration in rat and human plasma [41,42], it is required to modify $\mathrm{KO}$ to more active compounds by chemical or enzymatic transformations in order to enhance its water solubility and biological activity. Further studies on the bioavailability of $\mathrm{KO}$ and $\mathrm{KR}$ are required, and their in vivo efficacy studies would be needed to evaluate their potential PD-1/PD-L1 blocking effects in animal and human models. 


\section{Materials and Methods}

\subsection{Chemicals and Antibodies}

RPMI 1640 medium (\#SH30027.01), Dulbecco's Modified Eagle's Medium (DMEM, \#SH30243.01), F-12 Kaighn's Modification (\#SH30526.01) medium, fetal bovine serum (FBS, \#SH30084.3), 0.25\% trypsin-EDTA, and penicillin-streptomycin (\#SV30010) were purchased from Hyclone (South Logan, UT, USA). The human PD-1/PD-L1 Inhibitor Screening Assay Kit (\#72005), human PD-1 (hPD-1) neutralizing antibody (\#71120), and human PD-L1 (hPD-L1) neutralizing antibody (\#71213) were purchased from BPS Bioscience (San Diego, CA, USA). Kaempferitrin (KI), kaempferol (KO), and kaempferol 7-O-rhamnoside (KR) were purchased from ChemFaces (Wuhan, Hubei, China). PD-1/PD-L1 Inhibitor C1 (M60312-2s) was purchased from Xcess Biosciences Inc. (San Diego, CA, USA). The PD-1/PD-L1 Blockade Bioassay Kit was purchased from Promega (Fitchburg, WI, USA).

\subsection{Preparation of GHE}

Extracts of Geranii Herba (GHE) was obtained from the NIKOM (National Development Institute of Korean Medicine, Gyeongsan, Korea). GHE were freeze-dried to powder and then dissolved in DMSO with gentle shaking. After centrifugation at $480 \times g$ for $20 \mathrm{~min}$, the insoluble residues were removed and the supernatant was stored in a desiccator at $4{ }^{\circ} \mathrm{C}$ until the experiments [43].

\subsection{Competitive Enzyme-Linked Immunosorbent Assay (ELISA)}

To elucidate the effect of test inhibitors on PD-1/PD-L1 protein-protein interaction, PD-1/PD-L1 Competitive ELISA Screening Kits (BPS Bioscience Inc., San Diego, CA, USA) were used according to the supplier's instructions [27]. Briefly, recombinant hPD-L1 (BPS Bioscience, \#71104) was coated overnight at $1 \mu \mathrm{g} / \mathrm{mL}$ in phosphate-buffered saline (PBS) in 96-well plates (Corning Inc., New York, NY, USA). Plates were washed with PBS containing 0.1\% Tween (PBS-T), blocked for $1 \mathrm{~h}$ at room temperature with PBS containing $2 \%(w / v)$ BSA, and then washed again. Fifty microliters of $0.5 \mu \mathrm{g} / \mathrm{mL}$ biotinylated hPD-1 (BPS Bioscience, \#71109) was added to the wells, and the plates were incubated for $2 \mathrm{~h}$ at room temperature. After three washes in PBS-T, $50 \mu \mathrm{L}$ of $0.2 \mu \mathrm{g} / \mathrm{mL} \mathrm{HRP}$-conjugated streptavidin was added to each well, and the plates were incubated for $1 \mathrm{~h}$. After incubation, plates were washed three times in $0.1 \%$ PBS-T, and relative chemiluminescence was measured on a SpectraMax L Luminometer from Molecular Devices (San Jose, CA, USA).

\subsection{Cell Culture}

Jurkat $\mathrm{T}$ cells expressing firefly luciferase gene under the control of NFAT response elements with constitutive expression of human PD-1 (PD-1 Jurkat cells, effector cells) were obtained from BPS Bioscience (San Diego, CA, USA). PD-1 Jurkat cells were cultured in RPMI 1640 medium (Hycolone) supplemented with 10\% FBS, 1\% penicillin, and streptomycin. CHO-K1 cells constitutively expressing human PD-L1 and an engineered T cell receptor (TCR) activator (PD-L1/aAPC CHO-K1 cells, target cells) were obtained from BPS Bioscience (San Diego, CA, USA). PD-L1/aAPC CHO-K1 cells were incubated in F12/DMEM containing 10\% (v/v) heat-inactivated FBS and 1\% penicillin/streptomycin at $37{ }^{\circ} \mathrm{C}$ and $5 \% \mathrm{CO}_{2}$. Additionally, both cells were maintained in complete medium with Hygromycin B $(200 \mu \mathrm{g} / \mathrm{mL})$ and G418 (1 mg/mL) to select stable cell lines expressing the genetic constructs. For the experiments, Hygromycin B and G418 were not included in the medium.

\subsection{PD-1/PD-L1 Blockade Assay}

A PD-1/PD-L1 Blockade Bioassay kit was used according to the supplier's instructions (Promega, Fitchburg, WI, USA) with a slight modification [8]. Briefly, the PD-L1 aAPC/CHO-K1 cells were seeded in 96-well plates at a density of $5 \times 10^{4} /$ well in the complete culture medium. A few hours later, the medium was removed, and $1 \times 10^{5}$ PD-1 Jurkat cells were added to each well with test 
inhibitors. The cells were cultured for $24 \mathrm{~h}$ at $37^{\circ} \mathrm{C}$ and then lysed using the Bio-Glo ${ }^{\mathrm{TM}}$ Luciferase Assay System (Promega, Madison, WI, USA). After incubation for $15 \mathrm{~min}$, luminescence was quantified using a GloMax ${ }^{\circledR}$ Explorer Multimode Microplate Reader (Promega). Data are presented as fold to untreated control.

\subsection{Kinetic Analysis by Biolayer Interferometry (BLI Analysis)}

To measure the binding affinities and kinetic constants of test inhibitors, BLI analysis was conducted according to a previous report, with slight modifications [33]. Briefly, BLI on a BLItz system is a label-free analytic technology for biomolecular interactions by measuring interference patterns of light reflected from two surfaces [32]. After pre-equilibration of the SA BLI sensor (Pall FortéBio Corp., Menlo Park, CA, USA) in PBS buffer for 10 min, biotinylated human PD-1 (BioVision, Milpitas, CA, USA) and PD-L1 (Sino biological, Beijing, China) were fully loaded onto the sensors by immersion in $4 \mu \mathrm{L}$ of PD-1 and PD-L1 solution and then dissolved in PBS buffer to $50 \mu \mathrm{g} / \mathrm{mL}$. Test inhibitors for the kinetic analysis were prepared by 100-fold dilution of stock solution and dissolved in $100 \%$ $\operatorname{DMSO}(0,0.25,0.5$, and $0.75 \mathrm{mM})$ in PBS buffer. Binding kinetics were measured as follows: step 1, initial baseline in PBS buffer containing 1\% DMSO for $15 \mathrm{~s}$; step 2, association in $4 \mu \mathrm{L}$ of test inhibitors solution for $20 \mathrm{~s}$; and step 3, dissociation in PBS buffer containing 1\% DMSO for $20 \mathrm{~s}$. The kinetic constants were calculated using the BLItz Pro software by fitting the association and dissociation data to a 1:1 model. The equilibrium dissociation constant, $\mathrm{K}_{\mathrm{D}}$, was calculated as the dissociation constant $\left(\mathrm{k}_{\mathrm{d}}\right) /$ association constant $\left(\mathrm{k}_{\mathrm{a}}\right)$.

\subsection{In Silico Docking Simulation and Interaction Analysis}

The test inhibitors were docked onto the interaction space between PD-1 and PD-L1 (PDB code: $4 \mathrm{ZQK}$ ) retrieved from the Protein Data Bank (www.rcsb.org) and a previous report [34] using AutoDock Vina integrated with UCSF Chimera-alpha v1.13 [44]. The hydrophobic and hydrogen-bonding interactions between PD-L1 and each small molecule were analyzed using LigPlot+ v1.4.5 [45]. Amino acid residues involved in the interactions are indicated in red (hydrophobic interactions) and green (H-bonds).

\subsection{Statistical Analysis}

The data were expressed as mean \pm the standard error (S.E.) of the mean. Differences in the mean values between the treatment and control groups were analyzed by one-way analysis of variance with Dunnett's post-hoc test for multiple comparisons. GraphPad PRISM software ${ }^{\circledR}$ Version 5.02 (La Jolla, CA, USA) was used for analysis. $P$-values less than 0.05 were considered significant. Statistical differences are indicated using asterisks.

\section{Conclusions}

The present study indicated that $\mathrm{KO}$ and $\mathrm{KR}$ are active small molecule inhibitors against PD-1/PD-L1 interaction. Biochemical and in silico assays confirmed that KR may be a potential PD-1/PD-L1 inhibitor targeting both PD-1 and PD-L1. Therefore, we suggest that this PD-1/PD-L1 blockade of KR can be explained by its ability to interfere with the PD-1 and PD-L1 binding sites of their complex. Further studies should investigate whether KO and KR can act as PD-1/PD-L1 inhibitors in vivo in order to develop them as potent pharmaceuticals for immune checkpoint blockade therapy.

Supplementary Materials: The following are available online at http://www.mdpi.com/1422-0067/21/9/3239/s1, Figure S1: Effects of GHE on PD-1/PD-L1 protein interaction in a competitive ELISA, Figure S2, Effects of KO, KR, and $\mathrm{KI}$ on cell viability in PD-1 Jurkat cells and PD-L1/aAPC CHO-K1 cells, Figure S3, Effect of KO or KR on PD-1 expression in co-cultured PD-1 Jurkat cells and PD-L1 CHO-K1 cells, Figure S4, The binding of KO or KR against human PD-1 analyzed by SPR, Table S1: Estimated binding energy and interactions between amino acids of PD-1 protein and each compounds, Table S2: Estimated binding energy and interactions between amino acids of PD-L1 protein and each compounds. 
Author Contributions: Conceptualization, J.H.K., J.-G.C., Y.S.K., W.L., and H.-S.C.; Methodology, J.H.K., J.-G.C., Y.S.K., J.S., and H.-S.C.; Formal Analysis, J.H.K., J.-G.C., Y.S.K., J.-W.P., and J.S.; Investigation, J.H.K., J.-G.C., Y.S.K., W.L., E.J.L., J.-W.P., and J.S.; Resources, J.-G.C., Y.S.K., W.L., and H.-S.C.; Writing-original draft preparation, J.H.K.; Writing-Review and Editing, J.H.K., J.-G.C., Y.S.K., and H.-S.C.; Visualization, J.H.K.; Supervision, H.-S.C.; Project administration, H.-S.C.; Funding acquisition, H.-S.C. All authors have read and agreed to the published version of the manuscript.

Funding: This research was funded by the Korea Institute of Oriental Medicine (KIOM) from the Ministry of Science and ICT, Republic of Korea, grant number KSN2013230 and the Basic Science Research Program through the National Research Foundation (NRF) funded by the Ministry of Education (Grant number NRF-2018R1D1A1B07043760), Republic of Korea.

Acknowledgments: The National Development Institute of Korean Medicine (NIKOM) provided Korean herbal medicine sources for screening of PD-1/PD-L1 inhibitors. The authors especially thank Tae-In Kim in KIOM for help with drawing the chemical structures.

Conflicts of Interest: The authors declare no conflicts of interest.

\section{Abbreviations}

PD-1 Programmed cell death protein 1

PD-L1 Programmed death-ligand 1

TCR T-cell receptor

IFN- $\gamma \quad$ Interferon-gamma

NFAT Nuclear factor of activated T cells

IL-2 Interleukin 2

ICB Immune checkpoint blockade

SPR Surface Plasmon Resonance

BLI Biolayer Interferometry

$\mathrm{KO} \quad$ Kaempferol

KR Kaempferol-7-O-rhamnose

KI Kaempferitrin, kaempferol-3,7-dirhamnoside

\section{References}

1. Fife, B.T.; Pauken, K.E. The role of the PD-1 pathway in autoimmunity and peripheral tolerance. Ann. N. Y. Acad. Sci. 2011, 1217, 45-59. [CrossRef] [PubMed]

2. Guzik, K.; Tomala, M.; Muszak, D.; Konieczny, M.; Hec, A.; Blaszkiewicz, U.; Pustula, M.; Butera, R.; Domling, A.; Holak, T.A. Development of the Inhibitors that Target the PD-1/PD-L1 Interaction-A Brief Look at Progress on Small Molecules, Peptides and Macrocycles. Molecules 2019, 24, 2071. [CrossRef] [PubMed]

3. Oestreich, K.J.; Yoon, H.; Ahmed, R.; Boss, J.M. NFATc1 regulates PD-1 expression upon T cell activation. J. Immunol. 2008, 181, 4832-4839. [CrossRef] [PubMed]

4. Iwai, Y.; Hamanishi, J.; Chamoto, K.; Honjo, T. Cancer immunotherapies targeting the PD-1 signaling pathway. J. Biomed. Sci. 2017, 24, 26. [CrossRef] [PubMed]

5. Mizuno, R.; Sugiura, D.; Shimizu, K.; Maruhashi, T.; Watada, M.; Okazaki, I.M.; Okazaki, T. PD-1 Primarily Targets TCR Signal in the Inhibition of Functional T Cell Activation. Front. Immunol. 2019, 10, 630. [CrossRef] [PubMed]

6. Serfling, E.; Klein-Hessling, S.; Palmetshofer, A.; Bopp, T.; Stassen, M.; Schmitt, E. NFAT transcription factors in control of peripheral T cell tolerance. Eur. J. Immunol. 2006, 36, 2837-2843. [CrossRef]

7. Chen, L.; Flies, D.B. Molecular mechanisms of T cell co-stimulation and co-inhibition. Nat. Rev. Immunol. 2013, 13, 227-242. [CrossRef]

8. Skalniak, L.; Zak, K.M.; Guzik, K.; Magiera, K.; Musielak, B.; Pachota, M.; Szelazek, B.; Kocik, J.; Grudnik, P.; Tomala, M.; et al. Small-molecule inhibitors of PD-1/PD-L1 immune checkpoint alleviate the PD-L1-induced exhaustion of T-cells. Oncotarget 2017, 8, 72167-72181. [CrossRef]

9. Alsaab, H.O.; Sau, S.; Alzhrani, R.; Tatiparti, K.; Bhise, K.; Kashaw, S.K.; Iyer, A.K. PD-1 and PD-L1 Checkpoint Signaling Inhibition for Cancer Immunotherapy: Mechanism, Combinations, and Clinical Outcome. Front. Pharmacol. 2017, 8, 561. [CrossRef]

10. Borchers, A.T.; Hackman, R.M.; Keen, C.L.; Stern, J.S.; Gershwin, M.E. Complementary medicine: A review of immunomodulatory effects of Chinese herbal medicines. Am. J. Clin. Nutr. 1997, 66, 1303-1312. [CrossRef] 
11. Kwon, D.H.; Cheon, J.M.; Choi, E.O.; Jeong, J.W.; Lee, K.W.; Kim, K.Y.; Kim, S.G.; Kim, S.; Hong, S.H.; Park, C.; et al. The Immunomodulatory Activity of Mori folium, the Leaf of Morus alba L. in RAW 264.7 Macrophages In Vitro. J. Cancer Prev. 2016, 21, 144-151. [CrossRef] [PubMed]

12. Ma, H.D.; Deng, Y.R.; Tian, Z.G.; Lian, Z.X. Traditional Chinese Medicine and Immune Regulation. Clin. Rev. Allerg. Immu. 2013, 44, 229-241. [CrossRef] [PubMed]

13. Tan, B.K.H.; Vanitha, J. Immunomodulatory and antimicrobial effects of some traditional Chinese medicinal herbs: A review. Curr. Med. Chem. 2004, 11, 1423-1430. [CrossRef] [PubMed]

14. Ceci, C.; Tentori, L.; Atzori, M.G.; Lacal, P.M.; Bonanno, E.; Scimeca, M.; Cicconi, R.; Mattei, M.; de Martino, M.G.; Vespasiani, G.; et al. Ellagic Acid Inhibits Bladder Cancer Invasiveness and In Vivo Tumor Growth. Nutrients 2016, 8, 744. [CrossRef] [PubMed]

15. Coombs, M.R.; Harrison, M.E.; Hoskin, D.W. Apigenin inhibits the inducible expression of programmed death ligand 1 by human and mouse mammary carcinoma cells. Cancer Lett. 2016, 380, 424-433. [CrossRef] [PubMed]

16. Rawangkan, A.; Wongsirisin, P.; Namiki, K.; Iida, K.; Kobayashi, Y.; Shimizu, Y.; Fujiki, H.; Suganuma, M. Green Tea Catechin Is an Alternative Immune Checkpoint Inhibitor that Inhibits PD-L1 Expression and Lung Tumor Growth. Molecules 2018, 23, 2071. [CrossRef] [PubMed]

17. de Mejia, E.G.; Mazewski, C.; Luna-Vital, D.; Berhow, M. Black Lentil Aqueous Extract Attenuates Colitis-Associated Colon Carcinogenesis in Mice (OR04-06-19). Curr. Dev. Nutr. 2019, 3, OR04-06-19. [CrossRef]

18. Bao, F.; Bai, H.Y.; Wu, Z.R.; Yang, Z.G. Phenolic compounds from cultivated Glycyrrhiza uralensis and their PD-1/PD-L1 inhibitory activities. Nat. Prod. Res. 2019, 1-8. [CrossRef]

19. Han, Y.; Gao, Y.; He, T.; Wang, D.; Guo, N.; Zhang, X.; Chen, S.; Wang, H. PD-1/PD-L1 inhibitor screening of caffeoylquinic acid compounds using surface plasmon resonance spectroscopy. Anal. Biochem. 2018, 547, 52-56. [CrossRef]

20. Okuda, T.; Yoshida, T.; Mori, K. [Consitutents of Geranium thunbergii Sieb. et Zucc. II. Ellagitannins. (1) (author's transl)]. Yakugaku Zasshi 1975, 95, 1462-1466. [CrossRef]

21. Okuda, T.; Mori, K.; Murakami, R. [Constituents of Geranium thunbergii Sieb. et Zucc. VI. Difference of tannin activity caused by structural differences. (2). Colorimetry with methylene blue (author's transl)]. Yakugaku Zasshi 1977, 97, 1273-1278. [CrossRef]

22. Choi, H.J.; Choi, H.J.; Park, M.J.; Lee, J.Y.; Jeong, S.I.; Lee, S.; Kim, K.H.; Joo, M.; Jeong, H.S.; Kim, J.E.; et al. The inhibitory effects of Geranium thunbergii on interferon-gamma- and LPS-induced inflammatory responses are mediated by Nrf2 activation. Int. J. Mol. Med. 2015, 35, 1237-1245. [CrossRef]

23. Calderon-Montano, J.M.; Burgos-Moron, E.; Perez-Guerrero, C.; Lopez-Lazaro, M. A review on the dietary flavonoid kaempferol. Mini Rev. Med. Chem. 2011, 11, 298-344. [CrossRef]

24. Wang, J.; Fang, X.; Ge, L.; Cao, F.; Zhao, L.; Wang, Z.; Xiao, W. Antitumor, antioxidant and anti-inflammatory activities of kaempferol and its corresponding glycosides and the enzymatic preparation of kaempferol. PLoS ONE 2018, 13, e0197563. [CrossRef]

25. Xiufen, W.; Hiramatsu, N.; Matsubara, M. The antioxidative activity of traditional Japanese herbs. Biofactors 2004, 21, 281-284. [CrossRef]

26. Alonso-Castro, A.J.; Ortiz-Sanchez, E.; Garcia-Regalado, A.; Ruiz, G.; Nunez-Martinez, J.M.; Gonzalez-Sanchez, I.; Quintanar-Jurado, V.; Morales-Sanchez, E.; Dominguez, F.; Lopez-Toledo, G.; et al. Kaempferitrin induces apoptosis via intrinsic pathway in HeLa cells and exerts antitumor effects. J. Ethnopharmacol. 2013, 145, 476-489. [CrossRef]

27. Li, W.; Kim, T.I.; Kim, J.H.; Chung, H.S. Immune Checkpoint PD-1/PD-L1 CTLA-4/CD80 are Blocked by Rhus verniciflua Stokes and its Active Compounds. Molecules 2019, 24, 4062. [CrossRef]

28. Xia, A.; Zhang, Y.; Xu, J.; Yin, T.; Lu, X.J. T Cell Dysfunction in Cancer Immunity and Immunotherapy. Front. Immunol. 2019, 10, 1719. [CrossRef]

29. Tsai, H.F.; Hsu, P.N. Cancer immunotherapy by targeting immune checkpoints: Mechanism of T cell dysfunction in cancer immunity and new therapeutic targets. J. Biomed. Sci. 2017, 24, 35. [CrossRef]

30. Cai, J.; Wang, D.; Zhang, G.; Guo, X. The Role Of PD-1/PD-L1 Axis In Treg Development And Function: Implications For Cancer Immunotherapy. Onco Targets Ther. 2019, 12, 8437-8445. [CrossRef] 
31. Minchom, A.; Thavasu, P.; Ahmad, Z.; Stewart, A.; Georgiou, A.; O’Brien, M.E.R.; Popat, S.; Bhosle, J.; Yap, T.A.; de Bono, J.; et al. A study of PD-L1 expression in KRAS mutant non-small cell lung cancer cell lines exposed to relevant targeted treatments. PLoS ONE 2017, 12, e0186106. [CrossRef]

32. Zhai, W.; Zhou, X.; Du, J.; Gao, Y. In vitro assay for the development of small molecule inhibitors targeting PD-1/PD-L1. Methods Enzymol. 2019, 629, 361-381.

33. Boohaker, R.J.; Sambandam, V.; Segura, I.; Miller, J.; Suto, M.; Xu, B. Rational design and development of a peptide inhibitor for the PD-1/PD-L1 interaction. Cancer Lett. 2018, 434, 11-21. [CrossRef]

34. Zak, K.M.; Kitel, R.; Przetocka, S.; Golik, P.; Guzik, K.; Musielak, B.; Domling, A.; Dubin, G.; Holak, T.A. Structure of the Complex of Human Programmed Death 1, PD-1, and Its Ligand PD-L1. Structure 2015, 23, 2341-2348. [CrossRef]

35. Ganesan, A.; Ahmed, M.; Okoye, I.; Arutyunova, E.; Babu, D.; Turnbull, W.L.; Kundu, J.K.; Shields, J.; Agopsowicz, K.C.; Xu, L.; et al. Comprehensive in vitro characterization of PD-L1 small molecule inhibitors. Sci. Rep. 2019, 9, 12392. [CrossRef]

36. Musielak, B.; Kocik, J.; Skalniak, L.; Magiera-Mularz, K.; Sala, D.; Czub, M.; Stec, M.; Siedlar, M.; Holak, T.A.; Plewka, J. CA-170—A Potent Small-Molecule PD-L1 Inhibitor or Not? Molecules 2019, 24, 2804. [CrossRef]

37. Sung, Y.Y.; Yoon, T.; Yang, W.K.; Kim, S.J.; Kim, H.K. Anti-obesity effects of Geranium thunbergii extract via improvement of lipid metabolism in high-fat diet-induced obese mice. Mol. Med. Rep. 2011, 4, 1107-1113. [CrossRef]

38. Mazewski, C.; Kim, M.S.; Gonzalez de Mejia, E. Anthocyanins, delphinidin-3-O-glucoside and cyanidin-3-O-glucoside, inhibit immune checkpoints in human colorectal cancer cells in vitro and in silico. Sci. Rep. 2019, 9, 11560. [CrossRef]

39. Dabeek, W.M.; Marra, M.V. Dietary Quercetin and Kaempferol: Bioavailability and Potential Cardiovascular-Related Bioactivity in Humans. Nutrients 2019, 11, 2288. [CrossRef]

40. Yang, X.W.; Zhang, J.Y.; Xu, W.; Li, J.; Zhang, W.Q. [The biotransformation of kaempferitrin by human intestinal flora]. Yaо Xие Xие Baо 2005, 40, 717-721.

41. DuPont, M.S.; Day, A.J.; Bennett, R.N.; Mellon, F.A.; Kroon, P.A. Absorption of kaempferol from endive, a source of kaempferol-3-glucuronide, in humans. Eur. J. Clin. Nutr. 2004, 58, 947-954. [CrossRef]

42. Zhou, Z.; Wang, M.; Guo, Z.; Zhang, X. Pharmacokinetic evaluation of the interaction between oral kaempferol and ethanol in rats. Acta Pharm. 2016, 66, 563-568. [CrossRef]

43. Choi, J.G.; Kim, Y.S.; Kim, J.H.; Chung, H.S. Antiviral activity of ethanol extract of Geranii Herba and its components against influenza viruses via neuraminidase inhibition. Sci. Rep. 2019, 9, 12132. [CrossRef]

44. Pettersen, E.F.; Goddard, T.D.; Huang, C.C.; Couch, G.S.; Greenblatt, D.M.; Meng, E.C.; Ferrin, T.E. UCSF Chimera-A visualization system for exploratory research and analysis. J. Comput. Chem. 2004, 25, 1605-1612. [CrossRef]

45. Laskowski, R.A.; Swindells, M.B. LigPlot+: Multiple ligand-protein interaction diagrams for drug discovery. J. Chem. Inf. Model. 2011, 51, 2778-2786. [CrossRef]

(C) 2020 by the authors. Licensee MDPI, Basel, Switzerland. This article is an open access article distributed under the terms and conditions of the Creative Commons Attribution (CC BY) license (http://creativecommons.org/licenses/by/4.0/). 